Tohoku J. exp. Med., 1976, 118, 97-98

Short Report

\title{
Extraction of an Inhibitory Substance from Rat Liver on Rat Brain ATPase
}

\author{
Hiroshi Izumi, Makoto Hayakari, Shoryo Hayashi and \\ Hikaru Ozawa \\ Pharmaceutical Institute, Tohoku University, Aobayama, Sendai
}

IzUm, H., HayakaRi, M., Hayashu, S. and OzaWa, H. Extraction of an Inhibitory Substance from Rat Liver on Rat Brain ATPase. Tohoku J. exp. Med., 1976, 118 (1), 97-98 — A substance, which can be extracted from rat liver and partially purified, has been tested for its effect on the activity of ATPase in rat brain. This substance inhibited the ATPase activity significantly, and the inhibitory effect was not eliminated by the addition of catecholamines such as epinephrine and dopamine._ rat brain; ATPase

Adenosine triphosphatase (ATPase) activity associated with the subcellular fraction of brain has been the subject of intensive study. The presence of the factors regulating the activity of ATPase in rat brain have recently been suggested (Schaefer et al. 1972, 1974), although the purification and characterization of the factors have not yet been completed.

This finding led us to pursure the search for similar inhibitory principles in extract of other organs. We report here on the extraction from rat liver of a highly purified inhibitory substance.

Sprauge-Dawley female and male rats (CLEA, Japan, Inc.) weighing 250-300 $\mathrm{g}$ were used in these experiments. After animal was decapitated, the liver (15 g) was isolated and homogenized in 10 volumes of acetone-0.5 N HCl $(9: 1, v / v)$ using a Waring Blender at high speed for $45 \mathrm{sec}$. The mixture was stirred for $1 \mathrm{hr}$ at room temperature and then extracted with an equal volume of ether. The ether phase was evaporated at room temperature. The precipitate formed was removed by centrifuging at $105,000 \times \mathrm{g}$ for $30 \mathrm{~min}$. The supernatant was freezed-dried and the resulting material was dissolved in water. This material was fractionated by chromatography on a column $(30 \times 1.5 \mathrm{~cm})$ of Sephadex G-10 equilibirated with water. Samples of $2.5 \mathrm{ml}$ were collected and fractions $(13,14,15)$ were pooled and lyophilized. The lyophilized residue was rechromatographed by using a method of descending paper chromatography. The Rf value of the inhibitor in the solvent system of iso-propanol, pyridine and water $(4: 1: 4)$ is 0.73 . It shows a distinct U.V. spectrum. This material was extracted with water and its activity was detected by measuring the decrease in activity of ATPase when this substance was added to a suitable incubation medium.

The mitochondrial fraction was prepared by the procedure described below and used as the enzyme source of ATPase activity. Cerebral cortical grey matter was obtained from rats. Homogenates were prepared in 9 volumes of ice-cold $0.32 \mathrm{M}$ sucrose solution containing $3.0 \mathrm{mg} \mathrm{MgSO}_{4}$ and were centrifuged at $1,000 \times \mathrm{g}$ for $20 \mathrm{~min}$ to remove nuclei and cell debris. The mitochondrial fraction was recovered by centrifuging the $1,000 \times \mathrm{g}$ supernatant at $10,000 \times \mathrm{g}$ for $20 \mathrm{~min}$. All the procedures were carried out at $4^{\circ} \mathrm{C}$.

ATPase activity was assayed as described previously (Izumi et al. 1975).

As can be seen from Table 1, the activity of ATPase is reduced by the addition of the

Received for publication, September 22, 1975. 
TABLE 1. Effects of the inhibitory substance on ATPase in rat brain in the absence and presence of various biogenic amines

\begin{tabular}{lc}
\multicolumn{1}{c}{ Additions } & ATPase activity \\
\hline None & $47.60 \pm 0.35$ \\
Inhibitory substance (I.S) $(100 \mu \mathrm{l})$ & $26,00 \pm 0.25$ \\
Epinephrine & $55.20 \pm 0.14$ \\
Dopamine & $52.52 \pm 0.52$ \\
Phenethylamine & $48.55 \pm 0.11$ \\
I.S+epinephrine & $25.25 \pm 0.13$ \\
I.S +dopamine & $25.08 \pm 0.33$ \\
I.S + phenethylamine & $28.25 \pm 0.13$ \\
\hline
\end{tabular}

Enzymatic activities are expressed as $\mu \mathrm{g}$ of phosphate release $/ 350 \mu \mathrm{g}$ protein $/ 15$ min. Each value represents the mean of 3 experiments \pm s.e. The final concentration of respective amines added was $0.1 \mathrm{mM}$.

inhibitory substance to the assay system. We also tested the effects of catecholamines such as epinephrine and dopamine on the ATPase and also the effects of catecholamines plus inhibitory substance since catecholamines have been reported to abolish the inhibition by the soluble fraction of the rat brain (Schaefer et al. 1972). The addition of either epinephrine or dopamine resulted in a slight stimulation but phenethylamine did not, in agreement with those previously reported concerning catecholamine activation of $\mathrm{Na}^{+}-\mathrm{K}^{+}$-ATPase preparation from rat brain (Yoshimura 1973), although the extent of the stimulation is somewhat low. However, the inhibition by the inhibitory substance was not recovered at all by both catecholamines. These results suggest that the inhibitory substance from rat liver appears to be different from that studied in rat brain by Schaefer et al. $(1972,1974)$. At present no satisfactory explanation can be offered to this discrepancy.

The experimental results described in this report confirm the presence of an endogenous inhibitor of ATPase in rat liver. Whatever may be the mechanism responsible for the effects we have observed, it is important to establish the possible role in the regulation of ATPase in rat brain. Further investigation is necessary to determine whether this substance is present in other tissues and, conversely, this substance from rat liver inhibits ATPase from other tissues such as liver.

\section{References}

1) Izumi, H., Oyama, H. \& Ozawa, H. (1975) The stimulatory effect of the boiled supernatant on cyclic AMP formation in synaptosomes from rat cerebral cortex. Jap. J. Pharmacol., 25, 375-381.

2) Schaefer, A., Seregi, A. \& Komlos, M. (1974) Ascorbic acid-like effect of the soluble fraction of rat brain on adenosine triphosphatases and its relation to catecholamines and chelating agents. Biochem. Pharmacol., 23, 2257-2271.

3) Schaefer, A., Unyi, G. \& Pfeifer, A.K. (1972) The effects of a soluble factor and of catecholamines on the activity of adenosine triphosphatase in subcellular fractions of rat brain. Biochem. Pharmacol., 21, 2289-2294.

4) Yoshimura, K. (1973) Activation of Na-K activated ATPase in rat brain by catecholamine. J. Biochem., 74, 389-391. 